\title{
From the Editors: Conducting high impact international business research: The role of theory
}

Daniel C Bello

Area Editor

and

Tatiana Kostova

Consulting Editor

Correspondence:

DC Bello, J. Mack Robinson College of Business, Georgia State University, PO Box 3989, Atlanta, GA 30302, USA.

Tel: + 1404413 7658;

Fax: + 14044137276

\begin{abstract}
This editorial identifies a number of potential theory-related weaknesses in manuscripts, some more general in nature, others specific to international business (IB) research. It provides a brief critique and suggestions on how to overcome common problems in motivating research, framing contributions, reviewing literature, benefiting from the review process, and other issues of conceptual development. It also addresses questions particularly critical to IB research including multidisciplinarity, utilizing international context and enhancing theoretical impact. We conclude with specific guidelines on how to emphasize theory, thereby enhancing contribution and impact, in crafting and revising submissions to JIBS.

Journal of International Business Studies (2012) 43, 537-543. doi: I0. I057/jibs.20I2.14
\end{abstract}

Keywords: conceptual weaknesses; theory-related problems; multidisciplinarity; international context; theory development; author guidelines

\section{INTRODUCTION}

Frequently, manuscripts without major methodological flaws (no fatal problems of sampling, measurement, etc.) are rejected due to various conceptual problems, most of which are potentially correctable but are left unresolved by authors. Even papers that have made it successfully through the review process and have been published are often a source of disappointment as they do not have the impact that their authors were hoping for. The purpose of this editorial is two-fold. First, we discuss some major strategies helpful in strengthening or "correcting" conceptual weaknesses that, if left unaddressed, preclude manuscripts from publication even though they might be technically proficient. As is known in our field, presenting a logical and carefully developed theoretical model is a necessary condition for making it through the initial screening of a top-ranked academic journal. A significant improvement of the theory is also often required as part of an invited revision. Second, we offer suggestions on how to increase the potential impact of a paper through mindful conceptual work and through smarter positioning and framing. We believe that international business (IB) scholars are systematically missing opportunities to make a bigger contribution not only to the IB field but also to the organizational literature in general. We present 
a simple framework that should be useful in guiding authors as they strive to increase the impact of their work.

In what follows we address several key issues related to conceptual development of manuscripts in the context of both initial preparation for submission and revision after an invitation for resubmission. In addition to the more general theory-related problems, we identify some conceptual issues specific to IB research.

\section{GENERAL THEORY-RELATED ISSUES}

\section{Motivation and Justification}

Authors often fail to specify the motivation for their research, not explaining how and why the research is interesting, important to the field and differentiated from the extant literature. Readers should be able to understand very quickly the need for the research and appreciate the opportunity it provides for new insights and knowledge. Initial paragraphs should answer these basic questions: Why is this study/paper necessary? What is it about the phenomenon under investigation that is not understood? Where does the existing literature fall short on providing necessary explanations? How will this particular study add valuable insights and contributions? A paper can be motivated by a practical phenomenon that needs to be better understood and/or by the potential benefits of building upon an existing literature to strengthen it, broaden it or extend it in some new direction. Whatever motivates interest, the purpose of the research should be stated explicitly in the initial text. By the end of the introductory section, the reader should be convinced that this research is well justified.

We note several common mistakes in handling this issue. For example, a paper might claim that the existing literature does not provide answers to the research question being addressed. While this is a good start, we caution that identifying an apparent gap in the literature can never be more than a starting point for the more constructive and well-grounded development of a new argument. The fact that a question has not been previously studied is not a sufficient reason to address it. Authors should justify why the gap in current knowledge merits research attention and why it is important and interesting to study this particular question. Alternatively, a paper might suggest that a specific variation of a practical phenomenon has not been previously studied. This is particularly common in IB research, where scholars often find organizational phenomena that have not been explored in the international context (e.g., international R\&D teams vs R\&D teams in general). This could also be a good motivation for the research; however, the authors have to justify why the existing knowledge on the general phenomenon does not apply or needs to be modified or extended in the international context. What is the distinctiveness that the international aspect adds to the theoretical lens? Finally, in addition to being logically justified, the proposed research also needs to be interesting. Ours is a social science field, and our readership is strongly motivated by the surrounding world. Authors should try to capture the reader's attention by providing up front some startling statistics or anecdotes about the phenomenon that would further reinforce the logical justification for the research.

\section{Contribution and Framing}

Insufficient contribution to the subject area is perhaps the single most common reason for rejection at top scholarly journals. Research is published to disseminate new knowledge and communicate to others important findings, insights and ideas. Authors should specify how the research changes our understanding of the phenomenon, explaining the particular advances in theory development and other value-added aspects of research outcomes. For example, Whetten (1989) suggests authors should strive to make multiple contributions across the essential elements of theory, contributing to descriptions of their phenomena by altering the What (relevant constructs) and the How (causal connections) and to explanations by altering the Why (theoretical mechanisms, underlying logic) and the When (boundary conditions). In this way, authors state the particular way their research changes how we comprehend and reason about the phenomenon and advances our thinking about constructs and causal maps. By stating contributions explicitly, authors answer key questions about their research: What's new? Why so? So what?

A problem with regard to contribution could arise if the research question is too narrow and "small." While incremental research is still valuable as it adds to the common body of knowledge on a particular subject, broader, bigger and more novel questions tend to have a more notable contribution to the field. A common feature of highly impactful articles is their ability to specify how their concepts, ideas and findings are potentially useful beyond the narrow research domain of the particular study. 
Although there are limits to how much IB researchers can confidently cross over to other disciplines, and therefore a balance needs to be maintained, articulating multiple further applications is beneficial. It specifies the degree of generalization of the research to other areas of inquiry and as a result leads to a wider recognition and broader application of the work.

Other mistakes in this area are related to the way contribution is relayed in the paper. Authors can err on both sides, by doing too little to communicate the value of the research or by claiming too much. On the one hand, authors often fail to explicitly and clearly state the contribution of their work to theory and practice. In terms of theoretical contribution, it is important to clearly specify which particular literature the paper contributes to and how. Does the study confirm, expand or modify existing theory? Which theory or theories exactly are impacted by this research? Regarding practice, authors should be precise in specifying which particular targets can benefit from their findings managers in general, global managers, policymakers or some other groups? On the other hand, reviewers are often put off by abundant superlatives used to describe the contributions of a paper. It is important to be moderate and sensible in claiming contributions and to carefully substantiate each claim with specific evidence based on the results of the study. To achieve a balanced discussion of their own contribution, it is critical that authors reflect thoughtfully on the place their work takes in the existing literature. Unfortunately, this is often done in a superficial way, without proper recognition of previous contributions or a clear understanding of how the paper fits. Authors need to be able to demonstrate a breadth of knowledge and a depth of grounding in the most relevant literature in a parsimonious way. The clarity and precision with which the claimed contribution is formulated tends to be closely correlated with the authors' ability to establish tighter connections with the existing literature without creating an impression of redundancy and a lack of coherence in the list of references cited.

Finally, a common omission is failing to provide a thoughtful discussion of how the paper could serve as a foundation for future research. What are some of the important follow up and new research questions that could be generated based on the findings? Given that JIBS is intentionally multidisciplinary, it is also particularly important to propose questions that might motivate research in other cognate domains. Such reach outside of the
IB subject area facilitates the very important integration and incorporation of multiple disciplines as well as the academic standing of IB in social sciences.

\section{Literature and Positioning}

The contribution of a particular study is best appreciated when it is clearly positioned in the appropriate literature. It is critical for the reader to see how the study fits, builds on and contributes to the existing work on the topic. Although we rarely see a manuscript without a lengthy literature review section, there are still some common mistakes that authors should strive to avoid. Often, these sections are too descriptive and not sufficiently analytical. Because merely summarizing the literature adds little value, manuscripts benefit when extant literature is synthesized and integrated so new insights emerge, supporting a particular point of view and presaging the development of the theoretical model and formulation of research hypotheses. The objective of the literature review is much broader than simply providing an informed snapshot of the current work. It should be done in a way that helps the authors draw overall inferences from past studies as a group, identify overlooked antecedents and consequences, reveal various ambiguities and issues which have been inadequately addressed in prior research or reach insightful conclusions about prior research. Authors might also speculate on why different studies yield contradictory findings or employ diverse, possibly conflicting conceptualizations and definitions of constructs. MacKenzie (2003) notes that failure to reconcile ambiguous conceptualizations and findings in the extant literature often accounts for weak, inconsistent hypotheses, poor measurement of constructs and other serious problems in new research. Since many phenomena have rich, complex literature streams extending over many decades and disciplines, the literature review is not a trivial task. It requires authors to carefully assess and critique prior literature and also to carefully position their work in context. By doing so, authors can better articulate how and where their study fits into the existing body of knowledge and more effectively communicate how it extends current literature and improves our understanding of the phenomenon under study.

\section{Conceptual Development}

The heart of any research is its conceptual underpinnings in terms of theory or theoretical perspective taken, constructs, models and hypotheses. Without 
a clear theoretical foundation and convincing logic, research models risk mis- and under-specification, unexplained theoretical linkages and other problems that would most likely lead to rejection from a journal. The first common omission in this area is the lack of conceptual clarity in the construct definitions. IB research is particularly prone to this mistake as we tend to "borrow" constructs from underlying disciplines without always having a deep understanding of their conceptual core. In addition, we often introduce new constructs to capture novel phenomena, without fully doing the proper construct development work. Much more attention should be given to providing precise definitions for all constructs because underdevelopment and ambiguity create serious problems in developing the theoretical arguments, raise the risk of tautology and lead to confused operationalization and inadequate measurement.

Another fatal mistake is failure to adequately discuss the explanatory mechanisms justifying the proposed relationships between the constructs in the model. A recent editorial in JIBS (Thomas, Cuervo-Cazurra, \& Brannen, 2011) offers excellent ideas on how to address this issue. In addition to informed and disciplined theorizing on the general relationships in a model, authors should be careful about the nature of the proposed links to avoid mistakes like mixing direct, interactive, moderating and mediating effects. And hypotheses may lack compelling theoretical rationale, lack logical consistency and not represent a cohesive set with a strong conceptual theme.

Finally, a common problem we see is that many models are often too simplistic or trivial. As authors, we should push ourselves to develop more interesting and sophisticated hypotheses. Adding just one moderating variable or formally stating the obvious will not get us far. Commenting on this, Voss (2003) suggests one way to create interest is to be innovative in treatment of constructs and causal linkages: constructs in the domain of the phenomena can be defined, measured or analyzed in new ways and/or the conceptual system of relationships among constructs can be modified, mediated or moderated, revealing new complexity.

\section{Manuscript Revision}

In a more practical sense, authors can also better utilize the revision process. Many authors fail to extract the most benefit from the reviews and the invitation to revise, and they are not able to take their manuscripts to the next level. Dramatic improvements in theoretical and other weaknesses can occur by following the specific guidance of reviewers, focusing on basic theory-related issues and stepping back and reassessing the big picture. Because revision is "re-seeing" and "re-thinking," revising a manuscript involves a two-step process of new articulation and communication. Bello (2008: 386) notes, "First, re-writing involves a creative process of generation and discovery where key issues are better articulated and understood ... [Second], re-writing enhances communication more effectively conveying and revealing to others what the researcher now better understands." In this way, authors utilize reviewer and editor feedback to clarify their meaning and gain deeper insights into their research motivation, theoretical contribution, conceptual development and other elements with "correctable weaknesses." Since greater clarity of thought precedes improved communication, authors are urged to capitalize on an invitation to revise and resubmit to JIBS by carefully assessing and understanding conceptual problems and by revising in a way that better articulates and communicates the value of their research.

In addition to the more general theory-related issues discussed above, IB authors should also take into account a series of considerations that are specific and unique to our field of research. Below we discuss several such issues and offer suggestions of how to address them for producing higher impact work.

\section{IB-SPECIFIC THEORY-RELATED ISSUES}

\section{Multidisciplinarity}

The theoretical base of IB research is very broad and multidisciplinary. First, we have our own body of IB theories, frameworks, constructs and models (e.g., theories of internationalization of the firm, foreignness and others). Second, we draw heavily from other business and management areas such as strategic management, organizational behavior, human resource management, operations management, entrepreneurship and others. Third, we often extend our theoretical tools going all the way to base disciplines such as economics, sociology, political science, anthropology, psychology and others. Thus, our theoretical base is naturally not only multidisciplinary but also "layered," in that each type of framework in turn draws from the next one (e.g., psychology is the basis for much of the work in organizational behavior). This is both 
a blessing and a curse. On the one hand, such an approach provides opportunities for a rich theoretical examination that matches the complexity of IB phenomena. On the other hand, this multidisciplinary and layered theoretical base presents serious challenges for achieving conceptual clarity and rigor and even bigger challenges for integrating the various perspectives into a coherent theoretical story.

A major mistake observed in JIBS submissions is related to the use of multiple theories for the study of a given phenomenon. For example, scholars examining entry modes might employ, in the same paper, transaction cost economics, institutional theory, agency theory, resource-based view and others. The obvious advantage is that each theory provides a useful lens for looking at the phenomenon. However, this approach usually results in a laundry list of almost independent constructs, hypothesized relationships and explanatory mechanisms. Manuscripts following this path usually lack conceptual depth and rigor. An editorial by Cheng, Henisz, and Roth (2009) provides some excellent guidelines on how to integrate multiple disciplinary perspectives into a coherent theoretical base. Importantly, these authors stress that although the inherent complexity of IB phenomena can effectively be investigated through interdisciplinary research, valid applications of this approach must so thoroughly integrate and mix ideas and methods from two or more disciplines that the resulting product could not have been obtained by relying on a single discipline alone.

Another common problem is that IB scholars tend to adopt a very limited subset of ideas from the base disciplines and perspectives they use, without utilizing their theoretical richness and the many recent developments in those fields. The reasons are obvious - since we have limited capacity to follow many fields of research, we either return time after time to the same small subset with which we are familiar, or we rely on other IB scholars who have already brought these other literatures into our field. In both cases, we reiterate a somewhat simplistic and unsophisticated set of ideas from the underlying disciplines. Such skimming the surface of otherwise rich and rigorous theories does a real disservice to IB in that it limits our theorizing opportunities and also hurts the reputation of IB as a rigorous area of research. For example, there is a growing IB literature that employs the institutional theory perspective. However, a review of this literature shows that IB scholars use a rather limited and somewhat outdated set of institutional propositions (Kostova, Roth, \& Dacin, 2008). Drawing predominantly from neo-institutionalism, most of this work employs a much scaled down set of ideas revolving around institutional duality, that is, the pressures on MNEs from home and host countries, the related limited isomorphism and a few others. Mostly left out of the discussion are many other institutional ideas related to "old institutionalism," such as agency, power and politics, process of institutionalization, diffusion and so on, all of which could be extremely insightful and valuable for pushing IB research forward.

To address these issues, authors should limit the number of theoretical perspectives used to the most essential in light of the research question, as conceptual depth and rigor are much preferred to breadth; pay attention to the theoretical integration in case multiple perspectives are used exploring the complementary and/or interactive effects of the different theories; provide a more current and sophisticated application of borrowed theoretical perspectives by going to the foundational literatures and not relying only on their use in IB research.

\section{Utilizing Context}

The context in which we do our research - often the multinational company - provides unique opportunities for novel theory building. Yet it is rarely utilized for these purposes, as argued in a critical review article by Roth and Kostova (2003). The authors categorize published papers into three groups based on the main reason for which the MNE context has been used and critique the ways in which this scholarship has or has not contributed to advancing theory.

First is the study of MNE-specific phenomena, such as international joint ventures, foreign direct investment, entry modes, internationalization strategies, outsourcing, emerging markets and others. In this group authors tend to employ existing theories, use multiple theories, and take explanatory mechanisms from these theories. The international context is rarely considered in the model, and if it is, this is usually done in a simplistic way - by adding a context-specific variable such as national culture. The contribution of this set weighs more heavily on explaining the phenomenon than on theory development. A major omission in this type of research is that authors most often fail to specify the conceptual distinctiveness of the phenomenon under study and to utilize this distinctiveness for theory development. For example, what is theoretically different for international JVs 
compared to same country JVs? How are MNEs conceptually distinct from domestic firms? How are emerging market firms different from developed country firms? A commonly missed opportunity is that authors rarely tie their findings back to the dominant perspective they have used to further inform that perspective given the conceptual specificity of the MNE context. What have we learned based on the particular study that informs the theory itself?

Second is the set which validates or expands existing theories based on the substantial heterogeneity and complexity in the MNE context, including external or contextual complexity (e.g., multiple environments, diverse institutional and cultural settings), intra-organizational complexity (e.g., dispersed differentiated units, different management practices) and complexity at the individual level (e.g., variety of cognitive templates, values and beliefs). This work is potentially theoretically important as it challenges the assumptions and boundary conditions of theories typically developed in the West. It can help examine the generalizability of these theories and also expand them by including additional explanatory variables and stating moderating effects. To increase the theoretical impact of such research, authors should shift from an emphasis on theory extension to development of new theoretical insights based on the specific context. This is rarely done. A nice example of how this type of research could be used for theory extension is a recent meta-analysis paper (Carney, Gedajlovic, Heugens, Van Essen, \& Van Oosterhout, 2011) that tests the relationship between business group affiliation and performance. In addition to the base model, the authors test several hypotheses on conditions that moderate this relationship, including contextual variables like financial and labor market institutions. This constitutes a clear extension of the existing literature on business groups made possible by the use of the multiple-country context.

Third, some papers successfully use the MNE context for developing new theories and new theoretical insights. By studying IB phenomena and MNEs, authors have been able to re-specify and alter the basic logic of a given model. For example, in their study of team heterogeneity and performance, Earley and Mosakowski (2000) argue that the processes in cross-cultural teams cannot be sufficiently explained by existing theories from research in mono-cultural settings. These processes are instead heavily influenced by cultural identity theory, which fundamentally changes the predictions on this main relationship. The MNE context has also allowed scholars to develop new conceptualizations of organizations (e.g., Hedlund's "heterarchy," Bartlett and Ghoshal's "transnational") and to develop substantial theoretical additions to existing theories. For example, some MNE scholars applying the institutional perspective have been able to highlight the condition of institutional multiplicity and the mechanisms by which multiple institutional environments affect organizational units, and to provide novel conceptualizations of country institutional context, the effects of complex institutional embeddedness on knowledge transfer and sharing and so on.

\section{Theoretical Impact}

The problem is that the majority of IB articles fall under the first category identified in the previous section, some fall in the second, and very few in the third. The articles in the first two groups seldom make the effort to specify and convey the distinctive contribution to theory, as described above. The third approach to utilizing the MNE context and theory building is the one with the biggest potential impact on theory, yet is very rarely used. In that, IB research is missing huge opportunities to claim a major contribution not only to the IB field but also to management theory in general. Not surprisingly, the cross-referencing between the contributory disciplines and IB is severely asymmetric, with much more citation of main disciplines in IB papers than the other way around. Yet our unique context allows for major contributions that could feed back into the disciplines. Some of the directions that could help achieve these goals include, but are not limited to, the following: exploring the MNE as an extreme case for building new theory which then can be applied to any organization; proposing alternative explanatory mechanisms in light of the uniqueness of this context; addressing important questions that can be understood much better in MNEs; exploring the built-in plurality and tension in MNEs including centralized and decentralized decision making, worldwide integration and local autonomy of MNE operations, standardization vs differentiation in management practices and processes and so on; recognizing and exploring "combinative" phenomena that exist only in this context.

To develop a more impactful contribution in manuscripts, authors may find helpful the following simple suggestions. First, clearly identify the research 
objectives in terms of whether the approach is primarily focused on the study of international phenomena, on extending theory or on developing new theory, and if at all possible, strive for the higher level of contribution by pushing the theoretical model. A simple paragraph in the discussion section specifying findings that are generalizable to all types of contexts (e.g., any organization, not only MNE; any country context, not only emerging markets, etc.) vs those that are specifically valid for the international context make the nature of the contribution clear. Likewise, in developing the theoretical model for the study, authors should think carefully about the conceptual distinctiveness of the phenomenon due to its internationality, and based on that, try to develop more interesting ideas that would more clearly differentiate the work from previous non-international efforts. Finally, the biggest contributions will come from bold, novel theory-building efforts that push the research frontiers by fully utilizing the theoretically unique context of IB.

\section{GUIDELINES FOR AUTHORS}

To enhance the quality and impact of IB research, authors are encouraged to maximize the theoretical strength of their submissions. Before uploading a manuscript to the JIBS website, authors should assure themselves that key questions regarding their manuscript have been satisfactorily addressed:

- Is the research convincingly motivated and logically justified? Are the research objectives stated clearly?

\section{REFERENCES}

Bello, D. 2008. Writing, talking, and thinking! In N. Lee \& I. Lings (Eds), Doing business research: 385-386. Los Angeles: Sage.

Carney, M., Gedajlovic, E., Heugens, P., Van Essen, M., \& Van Oosterhout, H. 2011. Business group affiliation, performance, context and strategy: A meta-analysis. Academy of Management Journal, 54(3): 437-460.

Cheng, J., Henisz, W., \& Roth, K. 2009. From the Editors: Advancing interdisciplinary research in the field of international business: Prospects, issues and challenges. Journal of International Business Studies, 40(7): 1070-1074.

Earley, C., \& Mosakowski, E. 2000. Creating hybrid team cultures: An empirical test of transnational team functioning. Academy of Management Journal, 43(1): 26-49.

Kostova, T., Roth, K., \& Dacin, T. 2008. Institutional theory in the study of multinational corporations: A critique and new directions. Academy of Management Review, 33(4): 994-1007.
- Is prior literature synthesized and integrated? Are ambiguities and contradictions reconciled?

- Are theoretical perspectives treated with appropriate depth and rigor? Is the paper using the most current literature? Is the international context utilized to full effect, exploiting the conceptual distinctiveness of global phenomena and setting?

- Are the impacts of findings on theory building fully discussed for IB, and if appropriate, for base disciplines?

\section{CONCLUSION}

Using strong theory does not concern only individual authors and their chances of having their work published and recognized. It is also critical for the further advancement of the field of IB research as a whole. IB scholarship has made tremendous progress in the last few decades in establishing itself as a legitimate and respected area of social sciences. We have benefited from an incredibly interesting and dynamic global business environment which has motivated a continuous stream of fascinating questions. We have also leveraged knowledge from a number of primary disciplines for a more solid foundation for our research. We believe the field is now ready to take the next step and push harder the theoretical agenda, to individually and collectively capitalize on the unique context for developing major and novel contributions to organizational theories.

MacKenzie, S. 2003. The dangers of poor construct conceptualization. Journal of the Academy of Marketing Science, 31(3): 323-326.

Roth, K., \& Kostova, T. 2003. The use of the multinational corporation as a research context. Journal of Management, 29(6): 883-890.

Thomas, D., Cuervo-Cazurra, A., \& Brannen, M.-Y. 2011. From the Editors: Explaining theoretical relationships in international business research: Focusing on the arrows, NOT the boxes. Journal of International Business Studies, 42(9): 1073-1078.

Voss, G. 2003. Formulating interesting research questions. Journal of the Academy of Marketing Science, 31(3): 356-359.

Whetten, D. 1989. What constitutes a theoretical contribution? Academy of Management Review, 14(4): 490-495. 